\title{
Blood Vessels Segmentation in Retinal Fundus Image using Hybrid Method of Frangi Filter, Otsu Thresholding and Morphology
}

\author{
Wiharto $^{1}$, YS. Palgunadi ${ }^{2}$ \\ Department of Informatics, Universitas Sebelas Maret \\ Jl. Ir. Sutami, No. 36A, Surakarta Indonesia
}

\begin{abstract}
Diagnosis of computer-based retinopathic hypertension is done by analyzing of retinal images. The analysis is carried out through various stages, one of which is blood vessel segmentation in retinal images. Vascular segmentation of the retina is a complex problem. This is caused by non-uniform lighting, contrast variations and the presence of abnormalities due to disease. This makes segmentation not successful if it only relies on one method. The aims of this study to segment blood vessels in retinal images. The method used is divided into three stages, namely preprocessing, segmentation and testing. The first stage, preprocessing, is to improve image quality with the CLAHE method and the median filter on the green channel image. The second stage, segmenting using a number of methods, namely, frangi filter, 2D-convolution filtering, median filtering, otsu's thresholding, morphology operation, and background subtraction. The last step is testing the system using the DRIVE and STARE dataset. The test results obtained sensitivity $\mathbf{9 1 . 1 8 7 \%}$ performance parameters, $\mathbf{8 6 . 8 9 6 \%}$ specificity, and area under the curve (AUC) 89.041\%. Referring to the performance produced, the proposed model can be used as an alternative for blood vessel segmentation of retinal images.
\end{abstract}

Keywords-Segmentation; morphology; frangi filter; retinal; blood vessels

\section{INTRODUCTION}

Diagnosis of hypertensive retinopathy can be done by analyzing the retina of the eye. Analysis of the retina of the eye can be done using the image processing approach. The analysis was carried out to identify changes in retinal blood vessel patterns. Recognizing the pattern of blood vessels, a segmentation process is needed, which is to separate the blood vessels of the retina from the background. Segmentation results will clarify the pattern of retinal blood growth. The process of retinal image segmentation can be done using a number of existing segmentation methods. The segmentation method in image processing is divided into 4 classes, namely edge detection, thresholding-based, region-based and clustering-based [1]. Blood vessel segmentation in the retina is relatively difficult because the retinal image produced by the fundus camera has non-uniform lighting and contrast variations [2]. Another thing that causes difficulty in the process of segmentation is the presence of a disease so that an abnormality will emerge which may be similar to a blood vessel or other sign. This condition makes segmentation not only using one method but a combination of a number of methods.
A number of studies have segmented retinal blood vessels by various methods. Sabaz \& Atila [3] have segmented the retinal blood vessels by using a frangi filter. Frangi filter is a Hessian matrix based filter. The study succeeded in segmenting blood vessels, with the results of testing using the DRIVE dataset obtained $97.6 \%$ sensitivity, $72.6 \%$ specificity, and $86.04 \%$ accuracy. The resulting performance parameters have a high difference between sensitivity and specificity, so the AUC value is low. The performance of the study was relatively lower for specificity parameters if compared with the research conducted by Manikis et al. [4], which both used the hessian matric-based segmentation method. The performance of the study was $74.14 \%$, the specificity was $96.69 \%$ and the accuracy was $93.71 \%$, but the performance was also not balanced between the performance parameters of sensitivity and specificity, which resulted in a low AUC value.

The segmentation of blood vessel based on Hessian matric has also been done previously, namely by Ortiz et al. [5] and Ortiz et al. [6]. Both studies combined hessian matric and Gabor filters for segmentation, except that in both studies they did not measure the performance of the segmentation method used, but focused more on determining venous arterial ratios. The study of frangi filter was also used in the research of Oloumi \& Dhara [7]. The study not only uses frangi filtering, but also Gabor filters and a number of other methods. The segmentation method tested is a frangi filter, multiscale filter, gamma corrected green component, matched filter, and adaptive thresholding. The results of testing a number of methods, both single and hybrid, showed the highest specificity of $99.0 \%$, but the sensitivity was very low at $12.8 \%$ and the accuracy was only $87.8 \%$. This shows the ability of a number of methods combined with frangi filters has not been able to increase sensitivity. This condition makes the system unable to provide a balanced performance between sensitivity and specificity parameters, so the AUC value also becomes low.

The latest study was conducted by Khan et al. [8], which combines the frangi filter with the Vessel location map (VLM). The resulting performance, for parameter sensitivity was $73.0 \%$, specificity was $97.93 \%$ and accuracy was $95.8 \%$. The study was similar to that of Shahid \& Taj [2], which is a combination of the frangi filter with VLM. The results of these studies provide relatively the same performance. Similar research was also carried out by Nugroho et al. [9]. The research of Nugroho et al. [9] has proposed a segmentation 
method that combines frangi filters with morphological reconstruction. Tests performed using the DRIVE dataset show sensitivity of $72.13 \%$, specificity of $96.65 \%$ and accuracy of $94.5 \%$. The combination of frangi filter method with VLM, or frangi filter with morphology, has not been able to suppress differences in sensitivity performance parameters with specificity so that the AUC performance parameters produced are not optimal.

A number of studies on blood vessel segmentation using the frangi filter show that the frangi filter has not been able to work optimally when not combined with other methods. This is also supported in research conducted by Jothi \& Jayaram [10] [10]. The study concluded that the use of frangi filters with $3 \mathrm{D}$ hessian matrix has a fast computational process, but does not guarantee high accuracy in detecting blood vessels. Another thing is that the performance produced on the frangibased filter segmentation that has been carried out has higher specificity performance parameters than sensitivity, but with a big difference. A number of studies with such performance are carried out by [2], [4], [9], [11]-[17]. Another study is that sensitivity performance parameters are higher than specificity, but with high differences as well, as done by [3], [18]. The large difference between the two parameters makes the AUC parameter low.

Referring to a number of studies that have been conducted, this study proposes a combination of the frangi filter with a number of methods with the aim of improving performance. The performance improvement is indicated by the sensitivity and specificity being balanced so that the AUC value is better. The system model proposed in this study is segmentation using the hybrid method. The method consists of a frangi filter, 2D-convolution filter, FIR filter, otsu's thresholding, and morphology image. System performance is measured using sensitivity, specificity and AUC parameters.

\section{RESEARCH METHODS}

Research on the retinal blood vessel segmentation in the fundus image uses a combination of a number of methods. A combination of a number of methods with the aim of providing a balanced performance between sensitivity and specificity, so that the AUC value becomes higher. The proposed method is divided into 3 main processes, namely preprocessing, segmentation and performance analysis. Complete the proposed method as shown in Fig. 1. This study uses two datasets for testing, namely DRIVE [19] and STARE [20], which can be obtained by online.

\section{A. Preprocessing}

Preprocessing aims to improve the quality of retinal images. Preprocessing consists of three stages, first doing the process of separating retinal images into three channels, red, green and blue. The three channels are green channels which have the best quality, so the green channel is used for the next process. Second, improve the quality of retinal images using Contrast-limited adaptive histogram equalization (CLAHE) [21]. CLAHE is used to distribute color contrast. Third, to eliminate the amount of noise that appears, it is done using the median filter [22].

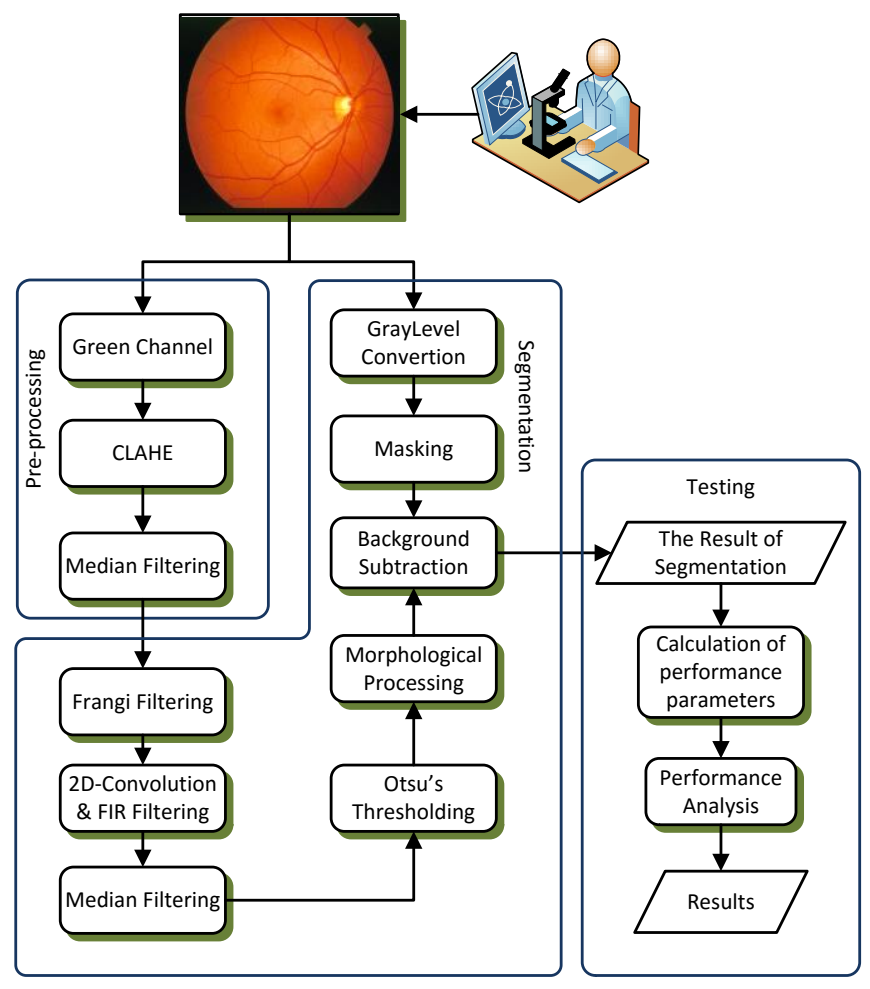

Fig. 1. Proposed Method.

\section{B. Frangi Filter}

The segmentation stages are a number of methods used, including the Frangi filter. Frangi filter serves to detect and improve the quality of blood vessels in retinal images. The retinal blood vessels have a wide diameter, the frangi filter will give the output of each pixel which has the maximum response when it detects blood vessels in the retina. The process of detecting frangi filter blood vessels using a hessian matrix kernel [3].

The Hessian matrix kernel in the frangi filter functions to analyze a function with more than one variable that is maximum or minimum under certain conditions. If a function is $f(x, y, z)$, the Hessian matrix can be formulated as shown in equation (1). The Hessian matrix shown in equation (1) is a Hessian matrix for functions of 3-dimensional [3].

$\mathbf{H f}(\mathrm{x}, \mathrm{y}, \mathrm{z})=\left[\begin{array}{ccc}\frac{\partial^{2} \mathrm{f}}{\partial \mathrm{x}^{2}} & \frac{\partial^{2} \mathrm{f}}{\partial \mathrm{x} \partial \mathrm{y}} & \frac{\partial^{2} \mathrm{f}}{\partial \mathrm{x} \partial \mathrm{z}} \\ \frac{\partial^{2} \mathrm{f}}{\partial \mathrm{y} \partial \mathrm{x}} & \frac{\partial^{2} \mathrm{f}}{\partial \mathrm{y}^{2}} & \frac{\partial^{2} \mathrm{f}}{\partial \mathrm{y} \partial \mathrm{z}} \\ \frac{\partial^{2} \mathrm{f}}{\partial \mathrm{z} \partial \mathrm{x}} & \frac{\partial^{2} \mathrm{f}}{\partial \mathrm{z} \partial \mathrm{y}} & \frac{\partial^{2} \mathrm{f}}{\partial \mathrm{z}^{2}}\end{array}\right]$

In the case of retinal images, it is enough to use 2 dimensions, namely $\mathrm{f}(\mathrm{x}, \mathrm{y})$, so that the Hessian matrix is as shown in equation (2) [23].

$\mathbf{H} f(x, y)=\left[\begin{array}{cc}\frac{\partial^{2} f}{\partial x^{2}} & \frac{\partial^{2} f}{\partial x \partial y} \\ \frac{\partial^{2} f}{\partial y \partial x} & \frac{\partial^{2} f}{\partial y^{2}}\end{array}\right]$ 
In this study $f(x, y)$ is a function with a Gaussian distribution. The Gaussian function for $2 \mathrm{D}$ can be shown in equation (3). The Hessian matrix is made using derivatives of equation (3).

$\mathrm{F}(\mathrm{x}, \mathrm{y})=\frac{1}{2 \pi \sigma^{2}} \mathrm{e}^{-\left[\left(\mathrm{x}-\mathrm{x}_{0}\right)^{2}+\left(\mathrm{y}-\mathrm{y}_{\mathrm{o}}\right)^{2}\right] /\left(2 \sigma^{2}\right)}$

The eigenvalue transformation in the Hessian matrix is used to obtain eigenvalues $\lambda_{1}$ and $\lambda_{2}$, while $\sigma$ is used to describe the scale of blood vessel repair. The filter response will be optimal if the scale $\sigma$ corresponds to the size of the blood vessel so that if the value of $\sigma$ is not correct it will reduce the performance of the frangi filter in detecting blood vessels. Frangi equation for finding the optimal retinal blood vessels, for two-dimensional images expressed in equations (4) $[18]$.

$V_{f}(s)= \begin{cases}0 & \text { if } \lambda_{2}>0 \\ \exp \left(-\frac{R_{B}}{\beta^{2}}\right)\left(1-\exp \left(-\frac{s^{2}}{2 c^{2}}\right)\right) & \text { otherwise }\end{cases}$

The $\beta$ and $c$ parameters in equation (4) are sensitivity parameters. The parameter value $R_{B}$ in equation (4) can be calculated by equation (5), while for the $S$ parameter it is calculated using equation (6) [18].

$R_{B}=\frac{\left|\lambda_{1}\right|}{\left|\lambda_{2}\right|}$

$S=\sqrt{\lambda_{1}^{2}+\lambda_{2}^{2}}$

\section{Convolution Filtering}

Convolution filtering is a $2 \mathrm{D}$ filter that is greatly influenced by the type of kernel it uses. In general, the convolution process can be shown in equation (7).

$\mathrm{G}(\mathrm{x}, \mathrm{y})=\sum_{\mathrm{s}=-\mathrm{a}}^{\mathrm{a}} \sum_{\mathrm{t}=-\mathrm{b}}^{\mathrm{b}} \mathrm{w}(\mathrm{s}, \mathrm{t}) \mathrm{f}(\mathrm{x}-\mathrm{s}, \mathrm{y}-\mathrm{t})$

where $g(x, y)$ is the convolution output, $f(x, y)$ is the original image, while $\mathrm{w}(\mathrm{s}, \mathrm{t})$ is the kernel used in the filter. The values of the parameters $s$ and $t$ are in the range of values $-\mathrm{a} \leq \mathrm{s} \leq \mathrm{a}$ and $-\mathrm{b} \leq \mathrm{t} \leq \mathrm{b}$. To improve the quality of blood vessels, the convolution filter is also integrated with a 2D FIR filter, with its type filter Circular averaging filter.

\section{Otsu's Thresholding}

The optimal threshold is the thresholding which results in the smallest possible segmentation error. The method that can be used to obtain optimal thresholding results is with Otsu [24]. Otsu thresholding has several advantages compared to other methods, namely computational speed and good capabilities when combined with other methods for performance improvement, and stable performance [25]. Otsu thresholding will automatically choose the optimal thresholding of the image, working on the assumption that the pixel of an image has two classes or a bimodal histogram. The Otsu method searches thoroughly by minimizing variance in the class [26]. The variance equation for each class is shown in equation (8).

$\sigma_{\mathrm{w}}^{2}(\mathrm{t})=\mathrm{q}_{1}(\mathrm{t}) \sigma_{1}^{2}(\mathrm{t})+\mathrm{q}_{2}(\mathrm{t}) \sigma_{2}^{2}(\mathrm{t})$
Where 1 and 2 show two classes, background, and foreground. The probability for each class and its variance can be calculated by equations (9-11).

$\mathrm{q}_{1}(\mathrm{t})=\sum_{\mathrm{i}=1}^{\mathrm{t}} \mathrm{P}(\mathrm{i})$

$\mathrm{q}_{2}(\mathrm{t})=\sum_{\mathrm{i}=\mathrm{t}+1}^{\mathrm{K}} \mathrm{P}(\mathrm{i})$

$\sigma_{1}^{2}(\mathrm{t})=\sum_{\mathrm{i}=1}^{\mathrm{t}}\left[\mathrm{i}-\mu_{1}(\mathrm{t})\right]^{2} \frac{\mathrm{P}(\mathrm{i})}{\mathrm{q}_{1}(\mathrm{t})}$

where $\mu_{1}(t)$ and $\mu_{2}(t)$ are the means of the class, which can be calculated by the equation (12-13).

$\mu_{1}(\mathrm{t})=\sum_{\mathrm{i}=1}^{\mathrm{t}} \frac{\mathrm{iP}(\mathrm{i})}{\mathrm{q}_{1}(\mathrm{t})}$

$\mu_{2}(t)=\sum_{i=t+1}^{K} \frac{i P(i)}{q_{2}(t)}$

Where the pixel value is in range of 0 to $\mathrm{K}$. Referring to Fig. 1, the use of the Otsu's method for thresholding is carried out after the frangi filtering process, which is then carried out by the morphology image process. In this study, the threshold value generated uses the Otsu method on a scale of $0-1$.

\section{E. Morphology Processing}

Morphology is an operation to change the shape structure contained in the image. Morphology operations involve two two-dimensional matrices. The first is a matrix of images that will be subject to morphology operations, while the second is the kernel matrix. This study uses three operations, namely closing, diagonal fill and Bridges unconnected pixels.

The closing operation is carried out using the mathematical model shown in equation (14). Referring to this equation, the closing operation is carried out by performing a dilation operation first and then followed by erosion operations [27].

A. $\mathrm{B}=(A \oplus B) \ominus B$

Diagonal fill operation is an operation to eliminate the 8connectivity background. The next operation Bridges unconnected pixels is bridging non-connected pixels, that is, specifying pixels worth 0 to 1 if they have two non-zero neighbors that are not connected. The binary morphology image operation aims to improve the quality of the output image of the Otsu thresholding, namely by reducing nonblood vessel pixels.

\section{F. Performance Analysis}

The performance of this research is measured by referring to the confusion matrix, as shown in Table I. The parameters used are sensitivity, specificity, accuracy, and area under the curve. These parameters can be shown in a formula in equations (15-17). Testing is done using two datasets, namely DRIVE and STARE. The each of dataset consists of 20 unsegmented images and 20 manually segmented images.

Sensitivity $=\frac{\mathrm{TP}}{\mathrm{TP}+\mathrm{FN}}$
Specificity $=\frac{\mathrm{TN}}{\mathrm{TN}+\mathrm{FP}}$

AUC $=\frac{\text { Sensitivity }+ \text { Specificity }}{2}[28]$ 
TABLE I. CONFUSION MATRIX

\begin{tabular}{|l|l|l|}
\hline \multirow{2}{*}{ Actual Class } & Predictive Class \\
\cline { 2 - 3 } & Positive & Negative \\
\hline Positive & TP (True Positive) & FN (False Negative) \\
\hline Negative & FP (False Positive) & TN (True Negative) \\
\hline
\end{tabular}

The model proposed in this study was implemented using Matlab R2014 software. The making and testing of the system are done by using a computer with Intel (R) Core (TM) i3$5005 \mathrm{U}$ CPU @ 2.00GHz 2.00 GHz, Memory 4.00GB, and using a 64-bit operating system.

\section{RESULTS AND DISCUSSION}

\section{A. Results}

Research carried out using the method as shown in Fig. 1 produced a number of outputs. First, the output for each process in preprocessing, as shown in Fig. 2. Fig. 2 is the test result using the DRIVE dataset. In Fig. 2(a) is the retinal image of the CLAHE process output and median filtering. Fig. 2(b) is the frangi filtering output and the final output of the segmentation method. The same test results using the STARE dataset, are shown in Fig. 3(a) and Fig. 3(b).

The next system output is the result of the proposed system performance measurement. Performance measurement is done by using two datasets, namely DRIVE and STARE. Performance parameters are measured by reference to equations (15-17), namely sensitivity, specificity, and AUC. The data used amounted to 20 retinal images, the results of segmentation using the proposed model were compared with the results of segmentation done manually by experts available in both datasets. Test results from 20 available retinal image data can be shown in Table II.

\section{B. Discussion}

The testing of the segmentation model proposed using the DRIVE and STARE datasets are able to provide performance as shown in Table II. Table II shows that for the DRIVE dataset it has a sensitivity that is greater than the specificity. This condition shows the system's average ability to detect pixels as blood vessels lower than the system's ability to detect pixels as background. In general, when referring to the AUC performance parameters indicate that the system is in a good category, that is, the AUC value is in the range of $80 \%-90 \%$ [29]. For the STARE dataset, the performance is relatively lower, because the characteristics of the STARE dataset between the background and blood vessels are finer than the DRIVE dataset, but the AUC value is still in the range of $80 \%-90 \%$.

The differences of the performance parameter sensitivity with relatively small specificity, when compared with a number of previous studies. Referring to Table III, the average difference between the two parameters is $23 \%$. The proposed system model is able to reduce sensitivity differences and specificity reaching $4.291 \%$. Sensitivity parameters in the proposed system, one of which is caused by the lack of accuracy in determining the combination of frangi filter parameter values, namely $\mathrm{c}$ and $\beta$ in equation (4). Incorrect combinations cause the values of the two parameters to be mutually opposite, or give a value that is not optimal. The performance generated in Table II is obtained by conducting a number of experiments, the combination of parameters $c$ and $\beta$, which is done manually. The results of a number of experiments obtained the best performance in the parameter value $c=106.580$ and $\beta=0.1058$. The choice of the best combination of $\mathrm{c}$ and $\beta$ values, it can be possible to develop using a number of computational intelligence algorithms, so that optimal performance will be obtained. Computational intelligence algorithms that can be used such as genetic algorithms, particle swarm optimization, and ant colony optimization.

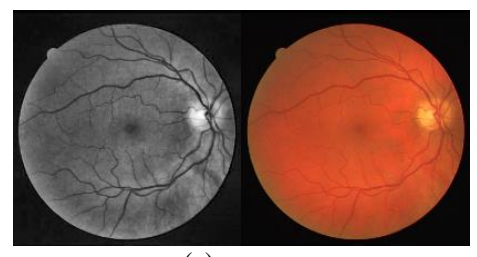

(a)

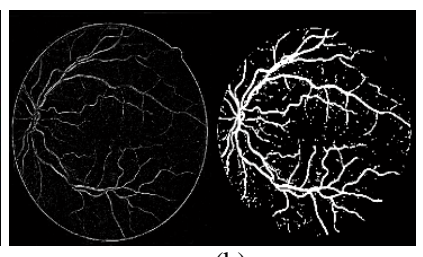

(b)
Fig. 2. Output System for DRIVE Dataset.

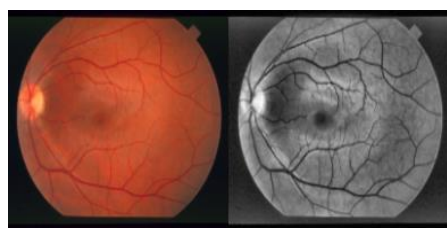

(a)

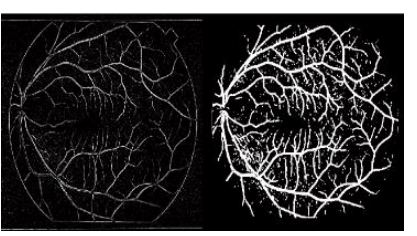

(b)
Fig. 3. Output System for STARE Dataset.

TABLE II. TeSting RESUlts USING DATASET

\begin{tabular}{|l|l|l|l|l|l|l|}
\hline \multirow{2}{*}{ No } & \multicolumn{5}{|l}{ DRIVE } & \multicolumn{2}{l|}{ STARE } \\
\cline { 2 - 7 } & SEN & SPE & AUC & SEN & SPE & AUC \\
\hline 1 & 88.649 & 92.714 & 90.681 & 89.651 & 84.393 & 87.022 \\
\hline 2 & 91.823 & 90.216 & 91.019 & 91.943 & 79.152 & 85.547 \\
\hline 3 & 89.839 & 86.331 & 88.085 & 86.145 & 87.844 & 86.994 \\
\hline 4 & 93.012 & 85.979 & 89.495 & 98.865 & 42.726 & 70.796 \\
\hline 5 & 93.824 & 84.013 & 88.918 & 86.914 & 92.068 & 89.491 \\
\hline 6 & 93.023 & 82.239 & 87.631 & 88.904 & 82.065 & 85.485 \\
\hline 7 & 92.422 & 84.721 & 88.571 & 85.025 & 92.139 & 88.582 \\
\hline 8 & 93.582 & 80.260 & 86.921 & 87.465 & 91.941 & 89.703 \\
\hline 9 & 94.450 & 82.215 & 88.332 & 88.914 & 93.385 & 91.149 \\
\hline 10 & 92.022 & 85.583 & 88.803 & 90.874 & 83.557 & 87.216 \\
\hline 11 & 89.141 & 86.249 & 87.695 & 85.832 & 95.664 & 90.748 \\
\hline 12 & 89.547 & 88.150 & 88.849 & 90.680 & 91.524 & 91.102 \\
\hline 13 & 90.580 & 85.201 & 87.891 & 90.303 & 85.932 & 88.118 \\
\hline 14 & 89.934 & 90.734 & 90.334 & 89.996 & 90.794 & 90.395 \\
\hline 15 & 88.409 & 90.040 & 89.224 & 87.798 & 88.191 & 87.995 \\
\hline 16 & 90.139 & 89.463 & 89.801 & 95.311 & 76.284 & 85.797 \\
\hline 17 & 91.049 & 85.129 & 88.089 & 87.049 & 93.018 & 90.033 \\
\hline 18 & 89.413 & 89.321 & 89.367 & 97.386 & 56.280 & 76.833 \\
\hline 19 & 90.279 & 90.822 & 90.551 & 97.551 & 47.418 & 72.484 \\
\hline 20 & 92.603 & 88.535 & 90.569 & 97.107 & 54.945 & 76.026 \\
\hline Mean & $\mathbf{9 1 . 1 8 7}$ & $\mathbf{8 6 . 8 9 6}$ & $\mathbf{8 9 . 0 4 1}$ & $\mathbf{9 0 . 6 8 6}$ & $\mathbf{8 0 . 4 6 6}$ & $\mathbf{8 5 . 5 7 6}$ \\
\hline & & & & & & \\
\hline
\end{tabular}


TABLE III. COMPARISON WITH PREVIOUS RESEARCH

\begin{tabular}{|l|l|l|l|l|}
\hline Author & SEN & SPE & SEN-SPE & AUC \\
\hline Aguirre-Ramos et al. [11] & 72.960 & 96.870 & 23.910 & 84.915 \\
\hline Akhavan et al. [30] & 72.520 & 97.330 & 24.810 & 84.925 \\
\hline Ali et.al. [31] & 78.180 & 96.880 & 18.700 & 87.530 \\
\hline Chakraborti et al. [12] & 72.050 & 95.790 & 23.740 & 83.920 \\
\hline Dash \& Bhoi [15] & 71.900 & 97.600 & 25.700 & 84.750 \\
\hline Frangi et al. [18] & 91.370 & 65.370 & 26.000 & 78.370 \\
\hline Jebaseeli et.al [32] & 70.040 & 99.800 & 29.760 & 84.920 \\
\hline Khan et al. [33] & 74.620 & 98.010 & 23.390 & 86.315 \\
\hline Manikis et al. [4] & 74.140 & 96.690 & 22.550 & 85.415 \\
\hline Memari et al. [14] & 76.100 & 98.100 & 22.000 & 87.100 \\
\hline Nugroho et al. [9] & 72.130 & 96.650 & 24.520 & 84.390 \\
\hline Oloumi et al. [7] & 87.600 & 91.800 & 4.200 & 89.700 \\
\hline Sabaz et al.[3] & 97.600 & 72.600 & 25.000 & 85.100 \\
\hline Shah et al. [13] & 72.050 & 98.140 & 26.090 & 85.095 \\
\hline Shahid \& Taj [2] & 73.000 & 97.930 & 24.930 & 85.000 \\
\hline Zhao et al. [34] & 73.540 & 97.890 & 24.350 & 85.715 \\
\hline Our proposed & $\mathbf{9 1 . 1 8 7}$ & $\mathbf{8 6 . 8 9 6}$ & $\mathbf{4 . 2 9 1}$ & $\mathbf{8 9 . 0 4 1}$ \\
\hline
\end{tabular}

The performance of the proposed hybrid segmentation model, when referring to the AUC parameter shows better capabilities than a number of studies that have been carried out, as shown in Table III. The proposed model has relatively the same performance compared to the model proposed by Oloumi et al. [7]. Unfortunately, the study which done by Oloumi et al. [7] used a combination of a number of methods which were relatively more numerous. The combined method is Multiscale Gabor filter, Frangi filter, gamma corrected green component, matched filter and line operator, with a fixed threshold. This means that good performance in the study of Oloumi et al. [7] must be supported by so many methods with high computation so that it also has a high complexity in its implementation and longer computation time.

The next comparison with the research conducted by Khan et al. [33] and Shahid \& Taj [2]. Both of these studies use a method that combines the frangi filter in segmentation. In this study, it produced almost the same performance, namely, AUC $86.315 \%$ and $85.00 \%$ for the DRIVE dataset. This performance is still lower compared to the proposed system. The same thing happened in a study conducted by Sabaz et al. [3], even parameter of specificity is lower when compared to the proposed segmentation model. Research by Sabaz et al. [3] has advantages in sensitivity parameters which can reach $97.6 \%$, but besides AUC the value of accuracy is also lower compared to the proposed system.

\section{CONCLUSIONS}

The segmentation model using a hybrid frangi filter, otsu's thresholding and morphology image are able to provide performance with an AUC parameter value of $89.041 \%$. or included in the good category. The proposed system specificity value, if seen the difference with the sensitivity parameter is not too high, which is only $4.291 \%$. This value is smaller than a number of studies that have been conducted. The model proposed, referring to the AUC value, can be an alternative method in segmenting retinal blood vessels. The performance of the proposed segmentation model can still increase when the values of the $c$ and $\beta$ parameters on the frangi filter are optimal values. Further research, in determining the parameter values can take advantage of a number of optimization algorithms, such as particle swarm optimization.

\section{ACKNOWLEDGMENT}

We would like to thank the Sebelas Maret University Indonesia. which has provided research grants of fundamentals with funding PNBP UNS. by contract number: $516 / \mathrm{UN} 27.21 / \mathrm{PP} / 2019$. The authors thank the anonymous referees for their constructive suggestions and comments which helped in the improvement of the presentation of the manuscript.

\section{REFERENCES}

[1] S. Samaddar and D. A. R. Reddy, "Comparative Study Of Image Segmentation Techniques On Chronic Kidney Diseases," Int. J. Pure Appl. Math., vol. 118, no. 14, pp. 235-239, 2018.

[2] M. Shahid and I. A. Taj, "Retracted: Robust Retinal Vessel Segmentation using Vessel's Location Map and Frangi Enhancement Filter," IET Image Process., vol. 12, no. 4, pp. 494-501, Apr. 2018.

[3] F. Sabaz and U. Atila, "Roi Detection And Vessel Segmentation In Retinal Image," ISPRS - Int. Arch. Photogramm. Remote Sens. Spat. Inf. Sci., vol. XLII-4/W6, pp. 85-89, Nov. 2017.

[4] G. C. Manikis et al., "An image analysis framework for the early assessment of hypertensive retinopathy signs," in The 3rd International Conference on E-Health and Bioengineering - EHB, Iaşi, Romania, 2011, pp. 1-6.

[5] D. Ortiz et al., "Support system for the preventive diagnosis of Hypertensive Retinopathy," in 32nd Annual International Conference of the IEEE EMBS, Buenos Aires, Argentina, 2010, pp. 5649-5652.

[6] D. Ortiz et al., "System Development for Measuring the Arterious Venous Rate (AVR) for the Diagnosis of Hypertensive Retinopathy," in Andean Region International Conference, Cuenca, Ecuador, 2012, pp. 53-56.

[7] F. Oloumi, A. K. Dhara, R. M. Rangayyan, and S. Mukhopadhyay, "Detection of Blood Vessels in Retinal Fundus Images," Comput. Sci. J. Mold., vol. 22, no. 2, pp. 155-185, 2014.

[8] K. B. Khan, Amir. A. Khaliq, A. Jalil, and M. Shahid, "A robust technique based on VLM and Frangi filter for retinal vessel extraction and denoising," PLOS ONE, vol. 13, no. 2, p. e0192203, Feb. 2018.

[9] H. A. Nugroho, R. A. Aras, T. Lestari, and I. Ardiyanto, "Retinal vessel segmentation based on Frangi filter and morphological reconstruction," in 2017 International Conference on Control, Electronics, Renewable Energy and Communications (ICCREC), Yogyakarta, 2017, pp. 181184.

[10] A. Jothi and S. Jayaram, "Blood Vessel Detection in Fundus Images Using Frangi Filter Technique," in Smart Innovations in Communication and Computational Sciences, vol. 670, B. K. Panigrahi, M. C. Trivedi, K. K. Mishra, S. Tiwari, and P. K. Singh, Eds. Singapore: Springer Singapore, 2019, pp. 49-57.

[11] H. Aguirre-Ramos, J. G. Avina-Cervantes, I. Cruz-Aceves, J. RuizPinales, and S. Ledesma, "Blood vessel segmentation in retinal fundus images using Gabor filters, fractional derivatives, and Expectation Maximization," Appl. Math. Comput., vol. 339, pp. 568-587, Dec. 2018.

[12] T. Chakraborti, D. K. Jha, A. S. Chowdhury, and X. Jiang, "A selfadaptive matched filter for retinal blood vessel detection," Mach. Vis. Appl., vol. 26, no. 1, pp. 55-68, Jan. 2015.

[13] S. A. A. Shah, T. B. Tang, I. Faye, and A. Laude, "Blood vessel segmentation in color fundus images based on regional and Hessian features," Graefes Arch. Clin. Exp. Ophthalmol., vol. 255, no. 8, pp. 1525-1533, Aug. 2017. 
[14] N. Memari, A. R. Ramli, M. I. B. Saripan, S. Mashohor, and M. Moghbel, "Retinal Blood Vessel Segmentation by Using Matched Filtering and Fuzzy C-means Clustering with Integrated Level Set Method for Diabetic Retinopathy Assessment," J. Med. Biol. Eng., Nov. 2018.

[15] J. Dash and N. Bhoi, "A thresholding based technique to extract retinal blood vessels from fundus images," Future Comput. Inform. J., vol. 2, no. 2, pp. 103-109, Dec. 2017.

[16] K. B. Khan, Amir. A. Khaliq, A. Jalil, and M. Shahid, "A robust technique based on VLM and Frangi filter for retinal vessel extraction and denoising," PLOS ONE, vol. 13, no. 2, p. e0192203, Feb. 2018.

[17] C. Zhu et al., "Retinal vessel segmentation in colour fundus images using Extreme Learning Machine," Comput. Med. Imaging Graph., vol. 55, pp. 68-77, Jan. 2017.

[18] A. F. Frangi, W. J. Niessen, K. L. Vincken, and M. A. Viergever, "Multiscale vessel enhancement filtering," in Medical Image Computing and Computer-Assisted Intervention - MICCAI'98, vol. 1496, W. M. Wells, A. Colchester, and S. Delp, Eds. Berlin, Heidelberg: Springer Berlin Heidelberg, 1998, pp. 130-137.

[19] J. Staal, M. D. Abramoff, M. Niemeijer, M. A. Viergever, and B. van Ginneken, "Ridge-Based Vessel Segmentation in Color Images of the Retina," IEEE Trans. Med. Imaging, vol. 23, no. 4, pp. 501-509, Apr. 2004.

[20] A. D. Hoover, V. Kouznetsova, and M. Goldbaum, "Locating blood vessels in retinal images by piecewise threshold probing of a matched filter response," IEEE Trans. Med. Imaging, vol. 19, no. 3, pp. 203-210, Mar. 2000.

[21] J. Ma, X. Fan, S. X. Yang, X. Zhang, and X. Zhu, "Contrast Limited Adaptive Histogram Equalization-Based Fusion in YIQ and HSI Color Spaces for Underwater Image Enhancement," Int. J. Pattern Recognit. Artif. Intell., vol. 32, no. 7, 2018.

[22] K. Noronha, K. T. Navya, and K. P. Nayak, "Support system for the automated detection of hypertensive retinopathy using fundus images," in International Conference on Electronic Design and Signal Processing (ICEDSP), Manipal, India, 2012, pp. 7-11.

[23] P. T. H. Truc, Md. A. U. Khan, Y.-K. Lee, S. Lee, and T.-S. Kim, "Vessel enhancement filter using directional filter bank," Comput. Vis. Image Underst., vol. 113, no. 1, pp. 101-112, 2009.
[24] N. Otsu, "A Threshold Selection Method from Gray-Level Histograms," IEEE Trans. Syst. Man Cybern., vol. 9, no. 1, pp. 62-66, Jan. 1979.

[25] W.-X. Kang, Q.-Q. Yang, and R.-P. Liang, "The Comparative Research on Image Segmentation Algorithms," in 2009 First International Workshop on Education Technology and Computer Science, Wuhan, Hubei, China, 2009, pp. 703-707.

[26] M. A. Ansari and S. K. Mahraj, "A Robust Methodfor Identification o fPaper Currency Using Otsu's Thresholding," presented at the 2018 International Conference on Smart Computing and Electronic Enterprise (ICSCEE), Shah Alam, Malaysia, 2018, pp. 1-5.

[27] F. Y. Shih, Image Processing and Mathematical Morphology Fundamentals and Applications. Boca Raton: CRC Press, 2009.

[28] E. Ramentol, Y. Caballero, R. Bello, and F. Herrera, "SMOTE-RSB *: a hybrid preprocessing approach based on oversampling and undersampling for high imbalanced data-sets using SMOTE and rough sets theory," Knowl. Inf. Syst., vol. 33, no. 2, pp. 245-265, Nov. 2012.

[29] F. Gorunescu, Data Mining Concepts,Models and Techniques, Intelligent Systems Reference Library. Berlin, Heidelberg: Springer, 2011.

[30] R. Akhavan and K. Faez, "A Novel Retinal Blood Vessel Segmentation Algorithm using Fuzzy segmentation," Int. J. Electr. Comput. Eng. IJECE, vol. 4, no. 4, pp. 561-572, 2014.

[31] A. Ali, W. M. D. Wan Zaki, and A. Hussain, "Retinal blood vessel segmentation from retinal image using B-COSFIRE and adaptive thresholding," Indones. J. Electr. Eng. Comput. Sci., vol. 13, no. 3, pp. 1199-1207, Mar. 2019.

[32] T. J. Jebaseeli, C. A. D. Durai, and J. D. Peter, "Segmentation of retinal blood vessels from ophthalmologic Diabetic Retinopathy images," Comput. Electr. Eng., vol. 73, pp. 245-258, Jan. 2019.

[33] K. BahadarKhan, A. A Khaliq, and M. Shahid, "A Morphological Hessian Based Approach for Retinal Blood Vessels Segmentation and Denoising Using Region Based Otsu Thresholding," PLOS ONE, vol. 11, no. 7, p. e0158996, Jul. 2016.

[34] Y. Qian Zhao, X. Hong Wang, X. Fang Wang, and F. Y. Shih, "Retinal vessels segmentation based on level set and region growing," Pattern Recognit., vol. 47, no. 7, pp. 2437-2446, Jul. 2014. 